\title{
Whither or Wither Microbicides?
}

\author{
Robert M. Grant ${ }^{1}$, Dean Hamer ${ }^{2}$ Thomas Hope $^{3}$, Rowena Johnston ${ }^{4}$, Joep Lange ${ }^{5}$, Michael \\ M. Lederman ${ }^{6}$, Judy Lieberman ${ }^{7}$, Christopher J Miller ${ }^{8}$, John P. Moore ${ }^{9}$, Donald E. \\ Mosier $^{10}$, Douglas D. Richman ${ }^{11}$, Robert T. Schooley ${ }^{12}$, Marty S. Springer ${ }^{13}$, Ronald S. \\ Veazey $^{14}$, and Mark A. Wainberg ${ }^{15}$
}

${ }^{1} \mathrm{~J}$. David Gladstone Institutes, University of California-San Francisco, San Francisco, CA 94518, USA ${ }^{2}$ National Cancer Institute, National Institutes of Health, Bethesda, MD 20817, USA

${ }^{3}$ Northwestern University, Chicago, IL 60611, USA ${ }^{4}$ Foundation for AIDS Research (amfAR), New York, NY 10005, USA ${ }^{5}$ Academic Medical Center, University of Amsterdam, Netherlands ${ }^{6}$ Case Western Reserve University, University Hospitals/Case Medical Center, Cleveland, OH 44106, USA ${ }^{7}$ Immune Disease Institute and Division of AIDS, Harvard Medical School, Boston, MA 02115, USA ${ }^{8}$ California National Primate Research Center, University of California-Davis, Davis, CA 95616, USA ${ }^{9}$ Weill Medical College of Cornell University, New York, NY 10065, USA ${ }^{10}$ Scripps Research Institute, La Jolla, CA 92037, USA ${ }^{11}$ San Diego Healthcare System and University of CaliforniaSan Diego, San Diego, CA 92093, USA ${ }^{12}$ University of California-San Diego, San Diego, CA 92093, USA ${ }^{13}$ Merck \& Co., Rahway, NJ 07090, USA ${ }^{14}$ Tulane National Primate Research Center, Covington, LA 70433, USA ${ }^{15}$ McGill University AIDS Centre, Jewish General Hospital, Montreal, Quebec H3T 1E2, Canada

\section{Abstract}

After disappointing results from all efficacy trials conducted to date, the field of microbicides research now faces substantial challenges. Poor coordination among interested parties and the choice of nonvalidated scientific targets for phase III studies have hampered progress and created mistrust about the use of microbicides as a method to prevent HIV-1 sexual transmission. Although new promising strategies are available, there will need to be serious reappraisals of how decisions are made to advance the next generations of candidates into clinical trials, and the use of appropriate animal models in this process will be critical.

The vaginal microbicide field faces yet another of its all-too-frequent crises after the outcome of the Carraguard efficacy trial, conducted by the Population Council in South Africa. This compound, a sulfated polysaccharide (polyanion), failed to demonstrate efficacy against HIV-1 vaginal transmission.

Almost simultaneously, the U.K. Microbicide Development Program reported that the highdose arm of the efficacy trial of another polyanion, PRO-2000, would be terminated immediately because there was no hope for demonstrating efficacy (1). It is a sign of the state of the field that there were sighs of relief when it became clear that Carraguard had not enhanced HIV-1 transmission rates, for this was the apparent outcome of the efficacy trial of Ushercell (cellulose sulfate), yet another polyanion, last year (2). With enhanced transmission occurring in the first ever microbicide efficacy trial, that of the detergent nonoxynol-9 (3), and probably at one of the trial sites of another detergent, Savvy (4), the track record of microbicide products in large-scale trials has been extremely poor. The failure of polyanions is not surprising because these compounds have limited potency in vitro, particularly against the most commonly transmitted strains of HIV-1, those that use the chemokine receptor CCR5 to enter cells $(5,6)$. Moreover, evidence is now emerging that cellulose sulfate can enhance HIV-1 infection in vitro, particularly of CCR5-using viruses (7). Because similar observations of polyanion- 
mediated enhancement of such viruses, both in vitro and in vivo, were made 15 to 20 years ago $(8,9)$, the subsequent testing of the polyanions in thousands of women raises concerns about the preclinical research that was performed on these microbicide candidates. Yet another detergent, sodium lauryl sulfate, is still being evaluated; the rationale for continuing this study is unclear. Questions must now be asked about the past and future directions of the microbicide field; the answers should help to frame the next phase of microbicide development.

\section{Why were detergents and polyanions selected for efficacy trials?}

The simple, and probably correct, answer is that decisions were based on the belief that preventing HIV-1 sexual transmission would be much easier to accomplish than turned out to be the case. Detergents disrupt HIV-1 efficiently in the test tube, and the polyanions have at least some antiviral activity in vitro $(3,5,6)$. Moreover, these compounds were cheap, available, and thought to be safe on the basis of in vitro studies. Phase 1 trials also revealed no major safety problems, although inflammation was observed in early tests of nonoxynol-9 $(3,10$, 11). Given the need to generate "momentum," and the lack, several years ago, of alternatives, key decision-makers in the microbicide field presumably believed that these products should be fast-tracked. Duplication of effort was an inevitable consequence when multiple funding agencies or institutions each felt the need to adopt its own polyanion candidate. Rather than comparing the different products in an animal model, identifying "the best," and coalescing behind a consensus proof-of-concept candidate, the various agencies entered at least five such compounds into phase II human trials, with three advancing to phase III. Perhaps a test of concept efficacy trial of a single polyanion might have been scientifically valid, but what happened was, and remains, difficult to justify. The competitive spirit is important in product development, but it can (and, in the case of the polyanion-based microbicides, did) cause an unacceptable waste of resources, both human and financial.

\section{Reverse transcriptase inhibitor-based microbicides: The next generation}

The next great hope of the microbicide field is that a reverse transcriptase inhibitor (RTI)based product might work. This concept is not without scientific merit, because the RTIs are generally potent, broadly active inhibitors both in vitro and in vivo $(12,13)$. Conducting an efficacy trial of an RTI-based microbicide seems justified, although we will raise some caveats below. Unfortunately, the structural problems in the microbicide field could reprise the errors of the past unless they are now resolved. Thus, there may not be a single efficacy trial, or even a pair of trials, of an RTI-based microbicide; there could be as many as four. The U.S. National Institutes of Health (NIH), the International Partnership for Microbicides (IPM), the Contraceptive Research and Development Agency, and the Population Council are all competing in this area. Rather than "The Great RTI Race," there needs to be a thorough, truly open and independent assessment of whether there should be large-scale testing of any RTIbased microbicide and, if so, a determination of which compound(s) should move forward and how many trials are needed to reach a conclusion. We recognize that there is merit in evaluating multiple RTIs at the preclinical stage and in small-scale human trials, particularly with the aim of identifying the most appropriate strategy for real-world use. For example, different compounds may have different physicochemical properties that facilitate formulation, something that could be assessed in nonhuman primates and in early-stage human trials. However, we believe there would have to be truly compelling, science-based reasons to advance more than one of these compounds into efficacy trials.

Currently, there is at best limited evidence from monkey models that topically applied RTIs can consistently protect from vaginal transmission, and most of the leading candidates have never been tested. A more substantial concern is that using RTIs for prevention might increase the spread of HIV-1 resistance, either because of their use by women who do not know their 
infection status, or because they select for the transmission of naturally resistant variants (14). There is precedence for resistance selection based on the use of nevirapine, a nonnucleoside RTI, to prevent mother-to-child transmission (15). What is uncertain, however, is the severity of the problem, which might in practice be minor and acceptable. The outcome may depend on whether topically applied RTIs can enter the circulation at levels high enough to drive resistance. This issue needs to be studied carefully in both nonhuman primates and humans before large-scale clinical trials are commenced. Conversely, the generation of RTIresistant viruses during therapy, an increasing problem now in Africa, could compromise the use of RTIs for prevention. There are, therefore, some risks associated with using RTI-based microbicides in any geographical area where broadly similar compounds form the mainstay of therapy (15).

\section{Alternative strategies}

Rather than gambling so many resources on testing multiple different RTIs, all of which have the same basic mechanism and viral target, the microbicide field should expand its focus to test a variety of molecules that attack HIV-1 at different points in its replication cycle. These include a new generation of antiretrovirals, the fusion or entry inhibitors that have already been proven to consistently and robustly protect macaques from vaginal transmission under highly stringent conditions $(12,16)$. Some of these compounds appear to have a high resistance barrier in vitro, and none is yet being used to treat HIV-1 infection in the developing countries where a microbicide is most needed.

Agreement could therefore be reached (at least in theory) between prevention- and treatmentbased organizations and companies to use entry inhibitors only for prevention in the developing world, with RTIs and protease inhibitors being reserved for therapy. As orally administered entry inhibitors do not yet appear to have any clinical advantages over other classes of drugs $(17,18)$, concerns about fair access to a diversity of antiretroviral agents may be trumped by the importance of preserving a plausible strategy for preventing infection. A similar agreement could be reached about integrase inhibitors, if these highly promising new compounds also prove to be useful as microbicides when tested in the macaque models. We are conscious, however, of the need to preserve some new drug classes for treatment of infection with multidrug-resistant viruses, because their emergence in the developing world must be anticipated. Moreover, the problems of resistance development during microbicide usage will apply to all classes of antiretroviral drugs, not just RTIs. These complex issues of prevention versus treatment priorities will require serious and informed discussions in the near future. Right now, these debates are not being held, with far-reaching decisions being made on an ad hoc basis. Persuading the pharmaceutical industry to restrict some of its drugs for HIV-1 prevention will not be easy, but some industry leaders have shown considerable vision in the past and may do so again given what is at stake.

\section{An expanded role for nonhuman primate models}

A critical issue for microbicides is the extent to which the nonhuman primate models have been ignored. This unfortunate situation reflects an attitude that only data derived from human trials are meaningful. The often-expressed fear is that a failed monkey experiment might lead to the rejection of a concept that could work in humans, but there also appears to be a reluctance to deal with inconvenient data that suggest a prominent microbicide strategy probably will not work in the real world. Any microbicide candidate being considered for human trials must demonstrate proof of both safety and efficacy in a macaque model, something that would be standard procedure in any product development program run by most major pharmaceutical companies. There are, at present, uncertainties about which particular nonhuman primate model is the best for testing prevention strategies, but until the potential value of these models 
becomes more widely accepted, the incentive and funding required to identify a consensus model will remain suboptimal. Success in a macaque model does not, of course, guarantee that the compound(s) will protect women from the far more daunting challenge posed by diverse HIV-1 strains, but failure to protect monkeys should be regarded as a serious sign that the compound lacks potency. Nonhuman primate challenge protocols must also be developed for assessing whether microbicide candidates might enhance susceptibility to infection. The development and widespread adoption of standardized preclinical testing programs is also needed. The perceived need to conduct large-scale trials should be replaced by the real need to test only the best agents and answer the most important questions, even if this means a reduction in the size and scope of the existing clinical trials program and the involvement of fewer funding agencies.

\section{Combination microbicides}

The microbicide field is still rooted in the "monotherapy" era, in that almost all planned or completed trials involve testing a single active compound. Anyone proposing to use monotherapy to treat HIV-1 infection would not be taken seriously nowadays, and the concept of multivalent vaccines is widely accepted. Yet the microbicide field continues to hope that a single compound will suffice to protect against the vast array of HIV-1 sequence variants that now circulate and are being sexually transmitted. This attitude seems incautiously optimistic. We recognize that logistical, practical, financial, and, perhaps above all, regulatory issues presently hinder the development of combination microbicides (19). Some of the regulatory concerns relate to the potential for adverse events when active agents that are individually safe are combined; this scenario seems unlikely with the newer antiretrovirals. There are also considerable scientific challenges in formulating more than one active agent in a single product. Nonetheless, given the overarching need for efficacy against diverse viral populations, these problems should now be tackled head on at the scientific, product development, and (in the case of FDA's outdated regulations) political levels.

We do not suggest that development of the more plausible, highly active single-agent microbicides should be delayed; we do, however, urge that all obstacles to the evaluation of combination products should be addressed now, and not just if and when single-agent products fail. We should also consider the possibility that an effective HIV-1 prevention program might require microbicides, vaccines, and/or pre-exposure prophylaxis, as well as behavioral science interventions, to be used together, not separately. The different arms of prevention science should cooperate, not compete as they do at present.

\section{The need to simplify microbicide usage}

One useful outcome of the Carraguard efficacy trial was an indication of what had long been suspected: that women actually use microbicides much less frequently than they report doing (44\% compared to $96 \%$ ) (20). But even a potent microbicide will be ineffective if it is not used consistently and correctly. This appears to be the case with products that must be used immediately before intercourse. Until more effective microbicide regimens are developed, potential solutions to the serious problem of poor adherence are formulations that need only be applied once daily (e.g., first thing in the morning) or sustained delivery devices such as inhibitor-loaded, vaginally inserted rings that gradually release active compounds for prolonged periods (21). These technologies are now being developed, notably by the IPM. In the meantime, is it worth further tests of formulations that women do not use often enough, particularly when evidence is lacking of their ability to provide durable protection in nonhuman primate models? 


\section{Is an effective microbicide possible?}

Several candidate microbicides can completely protect nonhuman primates from vaginal infection, which serves as a valuable proof of the overall concept $(12,16)$. The key problem areas seem now to be based in the practical issues of product development. A lack of expertise in this area might be the single biggest obstacle to success. It is a truism that pharmaceutical companies develop pharmaceutical products; nongovernmental organizations, academic scientists, and federal agencies rarely have the necessary experience. No major company has become directly involved in microbicide development, an important gap that may never be filled. One reason for the absence of the corporate sector relates to liability protection; solutions, including government-supported insurance, that apply to vaccines should be applied to other areas of prevention, including microbicides. In the absence of industry involvement, the nonprofit sector and various competing arms of the federal government have attempted to build or finance expertise in product development. This strategy is not working consistently well at present. It is arguable that NIH should now focus on its traditional role of supporting scientific discovery and that other federal agencies such as the U.S. Agency for International

Development should reconsider whether their involvement in microbicide research is helpful or just adds to the competition and confusion that now prevail. Whatever structure can be put in place for the nonprofit sector and federal agencies to work together to develop a microbicide product, the same hardheaded attitudes that prevail in the pharmaceutical industry must be adopted—wishful thinking should not be tolerated.

We believe it is time for some serious soul-searching within the microbicide field, with a substantial revision of its management, better and more accountable procedures for coordinating its major programs, and more attention paid to the underlying science. It will not be straightforward to coordinate and simplify the competing activities of multiple agencies, but if we concede failure before we even try, the problems of the past will persist and we will collectively fail to produce an effective microbicide. This would be a tragedy.

\section{References}

1. Microbicides Development Programme Update. www.mdp.mrc.ac.uk/downloads/MDP_statement_14Feb08_v1[1].2_FINAL.pdf

2. van de Wijgert JH, Shattock RJ. AIDS 2007;21:2369. [PubMed: 18025873]

3. Hillier SL, et al. J Acquir Immune Defic Syndr 2005;39:1. [PubMed: 15851907]

4. Feldblum PJ, et al. PLoS One 2008;3:e1474. [PubMed: 18213382]

5. Moulard M, et al. J Virol 2000;74:1948. [PubMed: 10644368]

6. Shattock RJ, Doms RW. Nat Med 2002;8:425. [PubMed: 11984565]

7. Tao W, Richards C, Hamer D. AIDS Res Hum Retroviruses 2008;24(7) epub ahead of print. 10.1089/ aid.2008.0043

8. Flexner C, et al. Antimicrob Agents Chemother 1991;35:2544. [PubMed: 1810188]

9. Meylan PR, Kornbluth RS, Zbinden I, Richman DD. Antimicrob Agents Chemother 1994;38:2910. [PubMed: 7695283]

10. Ward H, De La Court A, Kitchen V. Sex Transm Dis 1996;23:413. [PubMed: 8885074]

11. Stafford MK, et al. J Acquir Immune Defic Syndr Hum Retrovirol 1998;17:327. [PubMed: 9525433]

12. Klasse PJ, Shattock R, Moore JP. Annu Rev Med 2008;59:455. [PubMed: 17892435]

13. De Clercq E. Chem Biodivers 2004;1:44. [PubMed: 17191775]

14. Martinez J, Coplan P, Wainberg MA. Antiviral Res 2006;71:343. [PubMed: 16787667]

15. Duran AS, et al. AIDS 2007;21:199. [PubMed: 17197811]

16. Lederman MM, Offord RE, Hartley O. Nat Rev Immunol 2006;6:371. [PubMed: 16639430]

17. Saag, M., et al. 4th International AIDS Society Conference on HIV Pathogenesis, Treatment, and Prevention; 22 to 25 July 2007; Sydney. abstract WESS104 
18. Heera, J., et al. 15th Conference on Retroviruses and Opportunistic Infections; 3 to 6 February 2008; Boston. abstract 40LB

19. Coplan PM, Mitchnick M, Rosenberg ZF. Science 2004;304:1911. [PubMed: 15218130]

20. Cohen J. Science 2008;319:1026. [PubMed: 18292313]

21. Malcolm K, Woolfson D, Toner C, Lowry D. Biotechnol Genet Eng Rev 2004;21:81. [PubMed: 17017028]

\section{Biography}

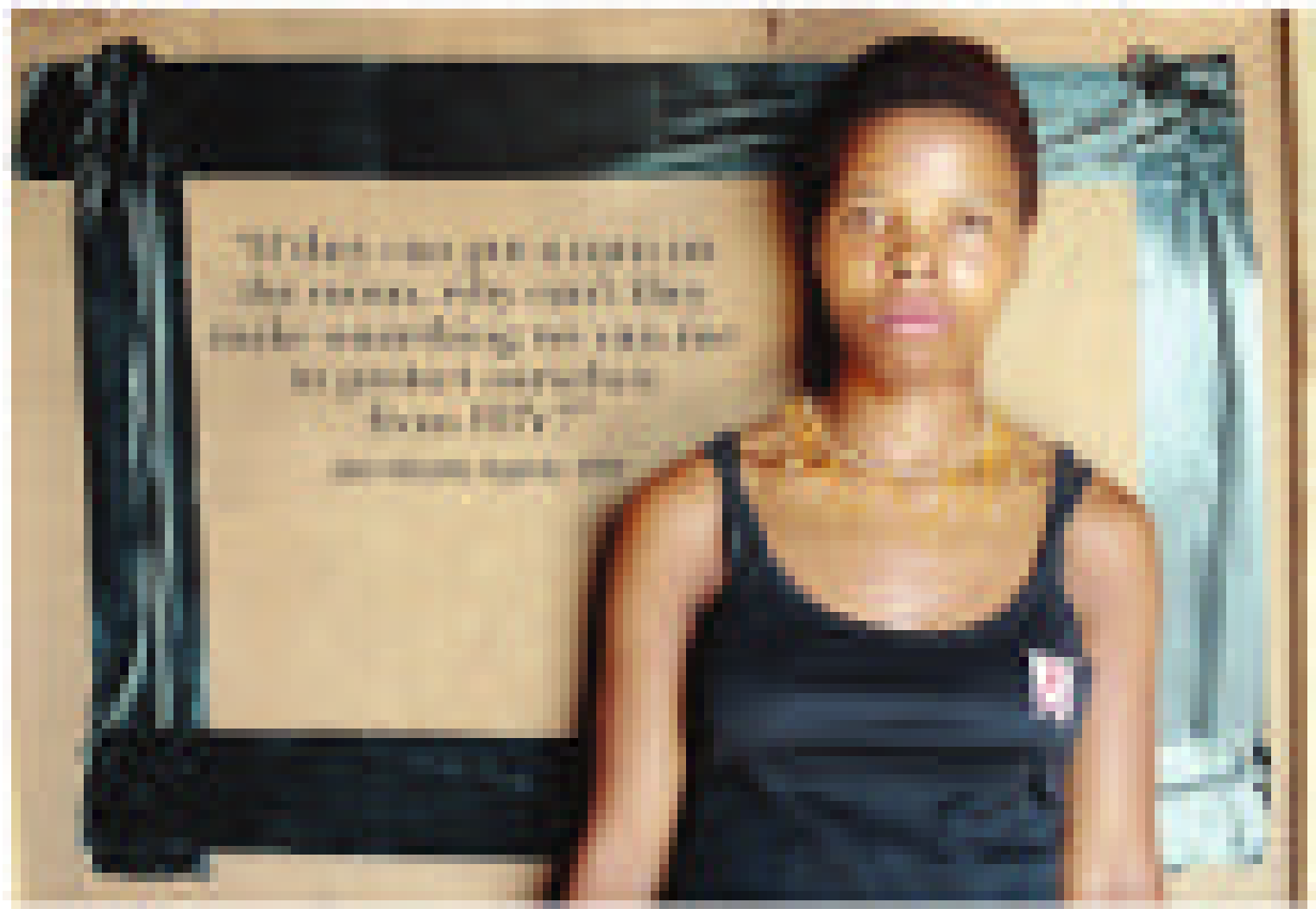

